\section{Ethnic variation among adolescent psychiatric in-patients with psychotic disorders}

\author{
JOVANKA TOLMAC and MATTHEW HODES
}

There is now strong evidence that the rates of psychiatric admission for psychosis are elevated in the British African-Caribbean population compared with the White British population (McGovern \& Cope, 1987; Commander et al, 1997). British AfricanCaribbean patients are also more likely to be detained under the Mental Heath Act 1983 (Pipe et al, 1991; Commander et al, 1997; Maden et al, 1999; Audini \& Lelliott, 2002) and in low-level and medium-secure psychiatric units (Koffman et al, 1997; Lelliott $e t$ al, 2001). Very few studies have been carried out investigating links between ethnic background and psychosis in the adolescent population. The only British study identified investigated child and adolescent psychiatric presentations for a defined area of London (Goodman \& Richards, 1995): second-generation African-Caribbean adolescents had significantly more psychotic disorders than the comparison group (3.4\% compared with $0.8 \%$ ) but the number of cases was small. We have performed a study to investigate whether Black adolescents are overrepresented in adolescent psychiatric units and, if they were, whether this is because of psychotic disorders. In view of the importance of pathways of care and experience of services, we also explored whether admission was more likely to be involuntary for the Black adolescent group.

\section{METHOD}

\section{Sample}

In this cross-sectional survey we included all young people aged 13-17 years who had a home address in the Greater London area and were psychiatric in-patients on our selected study day, 14 February 2001. Contact was established with 33 National Health Service and private sector adolescent and children's psychiatric units serving patients from Greater London; 114 wards in 35 adult mental health units in Greater London were also contacted, as well as a number of private psychiatric hospitals serving the London population. Identified young people were approached to take part in the study and written consent was obtained from them (and from their parents for the younger age group). For the young people who were difficult to access we collected information from the anonymised case summaries prepared and provided by the clinicians involved in their care.

\section{Measures}

We collected information from the case notes or from the anonymised case summaries using the data sheet designed for this study. Data were fully completed on 102 $(90 \%)$ young people but baseline demographic details were available for all adolescents. Psychiatric diagnoses according to DSM-IV diagnostic criteria (American Psychiatric Association, 1994) were assigned using the OPCRIT checklist and program (McGuffin et al, 1991). The OPCRIT system has been shown to have good reliability (Williams et al, 1996) and validity (Craddock et al, 1996) and has been used previously for adolescents with psychosis (Hollis, 2000). A sample of ten case summaries, assigned with the diagnosis of a psychotic disorder by the first rater (J.T.), was rated by the second rater (M.H.), who was blind to the results of the initial ratings. There was complete agreement between two ratings for diagnostic categories of psychotic disorders. In two cases there was agreement that the diagnosis was non-affective psychosis, but an assignment of different diagnostic subcategories was given by the raters. Eight adolescents who agreed to psychiatric interview $(15 \%$ of the 55 adolescents with psychosis) were interviewed using the Schedule for Affective Disorders and Schizophrenia for School Age Children (K-SADS; Chambers et al, 1985). There was complete agreement between OPCRIT and the K-SADS interview diagnoses on specific diagnostic categories.

We used UK Census 1991 ethnic categories for self-ascribed ethnicity recorded in the medical files. Because the numbers in each ethnic category were relatively small they had to be grouped into broader ethnic categories:

White: White British, White Irish, White Other

Black: Black British, Black Caribbean, Black African, Black Other

Asian: Indian, Pakistani, Bangladeshi, Asian Other 
Other: other ethnic groups and mixed ethnic origins.

We used the Census 1991 data (projections for the year 2001) for the total number of youngsters aged 13-17 years in different ethnic groups in Greater London. The Census 2001 data were not available at the time of writing this paper.

\section{Ethics}

We obtained approval from the London Multi-Centre Research Ethics Committee, as well as from the 40 relevant local research ethics committees.

\section{Data analysis}

The point prevalence of adolescents with psychosis who were psychiatric in-patients was calculated according to ethnic group. Odds ratios were estimated using logistic regression and calculated using the White adolescent group as the reference group. Confidence intervals were estimated at the 95\% level. Mantel-Haenszel analysis was used for estimates of the odds ratio and the relative risk. Differences in proportions were tested with Fisher's exact test and the $\chi^{2}$ test. Differences in the duration of hospital stay (regarded as a continuous variable) between different ethnic groups were established by analysis of variance (ANOVA). Results were analysed with SPSS for Windows (version 10) and STATA (version 5 , for windows).

\section{RESULTS}

There were 113 adolescents identified, aged 13-17 years, who were psychiatric inpatients on 14 February 2001. Ethnic and clinical diagnostic data are available on 110 adolescents (see Table 1).

A total of 95 patients $(84 \%)$ were in adolescent psychiatric units and 18 young people $(16 \%)$ were in-patients on adult psychiatric wards. The mean age was $\mathbf{1 6 . 2}$ years. Of all the in-patients, 55 (49\%) were admitted for assessment and treatment of either definite or suspected psychotic disorder. They were predominantly male and older adolescents: 38 males $(70 \%) v .17$ females $(30 \%)$; $40(73 \%)$ in-patients aged 16 or 17 years. Their demographic details are summarised in Table 2.

In this group of young people with clinical features of psychotic disorders, complete information was available on 51 young people and therefore OPCRIT could

Table I All psychiatric adolescent in-patients (clinical diagnosis/ethnicity/gender)

\begin{tabular}{|c|c|c|c|c|c|c|c|c|}
\hline \multirow[t]{2}{*}{ Ethnicity } & \multirow[t]{2}{*}{ Gender } & \multicolumn{7}{|c|}{ Diagnosis } \\
\hline & & $\begin{array}{c}\text { Psychotic } \\
\text { disorders } \\
\text { (\%) }\end{array}$ & $\begin{array}{c}\text { Affective } \\
\text { disorders } \\
\text { (\%) }\end{array}$ & $\begin{array}{c}\text { Eating } \\
\text { disorders } \\
\text { (\%) }\end{array}$ & $\begin{array}{c}\text { Anxiety } \\
\text { disorders } \\
\text { (\%) }\end{array}$ & $\begin{array}{c}\text { Conduct } \\
\text { disorders } \\
\text { (\%) }\end{array}$ & $\begin{array}{c}\text { Other } \\
\text { (\%) }\end{array}$ & $\begin{array}{l}\text { Total } \\
\text { (\%) }\end{array}$ \\
\hline \multirow[t]{3}{*}{ White } & Male & $16(59)$ & I (4) & 0 & $3(I I)$ & $2(7)$ & $5(19)$ & $27(100)$ \\
\hline & Female & $9(23)$ & I5 (38) & $8(2 I)$ & $2(5)$ & I (3) & $4(10)$ & $39(100)$ \\
\hline & Total & $25(38)$ & $16(24)$ & $8(12)$ & $5(8)$ & $3(5)$ & $9(14)$ & $66(100)$ \\
\hline \multirow[t]{3}{*}{ Black } & Male & $16(100)$ & 0 & 0 & 0 & 0 & 0 & $16(100)$ \\
\hline & Female & $3(60)$ & $2(40)$ & 0 & 0 & 0 & 0 & $5(100)$ \\
\hline & Total & $19(90)$ & $2(10)$ & 0 & 0 & 0 & 0 & $21(100)$ \\
\hline \multirow[t]{3}{*}{ Asian } & Male & $4(80)$ & 0 & 0 & 0 & I (20) & 0 & $5(100)$ \\
\hline & Female & $3(50)$ & 0 & I (I7) & $2(33)$ & 0 & 0 & $6(100)$ \\
\hline & Total & $7(64)$ & 0 & I(9) & $2(18)$ & I (9) & 0 & II (100) \\
\hline \multirow[t]{3}{*}{ Other } & Male & $2(50)$ & I (25) & 0 & I (25) & 0 & 0 & $4(100)$ \\
\hline & Female & $2(25)$ & $2(25)$ & $2(25)$ & $I(12)$ & 0 & $I(12)$ & $8(100)$ \\
\hline & Total & $4(33)$ & $3(25)$ & $2(17)$ & $2(17)$ & 0 & I (8) & $12(100)$ \\
\hline Total & & $55 \quad(50)$ & 21 (19) & II (I0) & $9(8)$ & $4(4)$ & 10 & $110(100)$ \\
\hline
\end{tabular}

be used to ascertain DSM-IV diagnoses: 23 $(45 \%)$ had diagnoses of schizophrenia spectrum disorders, $9(18 \%)$ had diagnoses of affective psychotic disorders (including schizoaffective disorders) and 20 $(37 \%)$ were diagnosed with a psychotic disorder not otherwise specified. All the cases diagnosed with psychotic disorders by the clinicians have also received an
OPCRIT DSM-IV diagnosis of a psychotic disorder.

Ethnic representation in in-patient units is different from the expectations based on the representation in the population of Greater London in this age group (Table 3). Young people in the Black group (Black Caribbean, Black African and Black British) were overrepresented in the in-patient

Table 2 Socio-demographic details of the 55 adolescents with psychosis

\begin{tabular}{|c|c|c|c|c|c|}
\hline & $\begin{array}{c}\text { White } \\
n(\%)\end{array}$ & $\begin{array}{l}\text { Black } \\
n(\%)\end{array}$ & $\begin{array}{c}\text { Asian } \\
n(\%)\end{array}$ & $\begin{array}{c}\text { Other } \\
n(\%)\end{array}$ & $\begin{array}{l}\text { Total } \\
n(\%)\end{array}$ \\
\hline \multicolumn{6}{|l|}{ Gender } \\
\hline Male & $16(64 \%)$ & $16(84 \%)$ & 4 (57\%) & $2(50 \%)$ & $38(70 \%)$ \\
\hline Female & $9(36 \%)$ & $3(16 \%)$ & $3(43 \%)$ & $2(50 \%)$ & $17(30 \%)$ \\
\hline \multicolumn{6}{|l|}{ Age (years) } \\
\hline $13-15$ & $7(28 \%)$ & $6(32 \%)$ & I (14\%) & I (25\%) & $15(27 \%)$ \\
\hline $16-17$ & 18 (72\%) & $13(68 \%)$ & 6 (86\%) & 3 (75\%) & $40(73 \%)$ \\
\hline \multicolumn{6}{|l|}{ Country of birth } \\
\hline UK & $22(88 \%)$ & $6(32 \%)$ & 6 (86\%) & $2(50 \%)$ & $36(65 \%)$ \\
\hline Outside UK & $3(12 \%)$ & $12(63 \%)$ & I (14\%) & $2(50 \%)$ & $18(33 \%)$ \\
\hline No information & 0 & I (5\%) & 0 & 0 & I $(2 \%)$ \\
\hline \multicolumn{6}{|l|}{ Living arrangement } \\
\hline With family & 21 (84\%) & $13(68 \%)$ & 7 (100\%) & $4(100 \%)$ & $45(82 \%)$ \\
\hline Without family & 2 (8\%) & $4(21 \%)$ & 0 & 0 & $6(11 \%)$ \\
\hline No information & $2(8 \%)$ & $2(11 \%)$ & 0 & 0 & $4(7 \%)$ \\
\hline \multicolumn{6}{|l|}{ Social class } \\
\hline I-III(n) & $9(36 \%)$ & $3(16 \%)$ & $2(29 \%)$ & $2(50 \%)$ & $16(29 \%)$ \\
\hline III(m)-V & $4(16 \%)$ & $3(16 \%)$ & I (14\%) & I (25\%) & $9(16 \%)$ \\
\hline Unemployed/student & $8(32 \%)$ & $13(68 \%)$ & 4 (57\%) & 0 & $25(46 \%)$ \\
\hline No information & $4(16 \%)$ & 0 & 0 & I (25\%) & $5(9 \%)$ \\
\hline
\end{tabular}


Table 3 Ethnic representation of the 55 adolescents with psychosis

\begin{tabular}{lccccccc}
\hline & $\begin{array}{c}\text { Total population } \\
\text { in London }\end{array}$ & $\begin{array}{c}\text { In-patients with diagnosis } \\
\text { of psychotic disorder }\end{array}$ & $\begin{array}{c}\text { Rate per } 10000 \\
(95 \% \mathrm{Cl})\end{array}$ & $\begin{array}{c}\text { Odds } \\
\text { ratio }\end{array}$ & $\begin{array}{c}\text { Standard } \\
\text { error }\end{array}$ & $95 \% \mathrm{Cl}$ \\
\hline White & $300913(64.5 \%)$ & $25(45 \%)$ & $0.831(0.638-1.082)$ & 1.0 & N/A & N/A \\
Black & $62348(13.4 \%)$ & $19(35 \%)$ & $3.048(2.341-3.968)$ & 3.7 & $1 . I$ & $2.0-6.7$ \\
Asian & $81697(17.5 \%)$ & $7(13 \%)$ & $0.857(0.658-1.116)$ & 1.0 & 0.4 & $0.4-2.4$ \\
Other & $21478(4.6 \%)$ & 4 & $(7 \%)$ & $1.863(1.431-2.426)$ & 2.2 & 1.2 & $0.8-6.4$ \\
Total & $466436(100 \%)$ & $55(100 \%)$ & $1.178(0.905-1.534)$ & N/A & N/A & N/A \\
\hline
\end{tabular}

$\mathrm{N} / \mathrm{A}$, not available.

adolescent psychotic population compared with the White group (odds ratio=3.7 after adjusting for gender). The relative risk for being in an in-patient unit is greater for the older Black adolescents $(4.7$; 95\% CI 2.59.0) than for younger adolescents and is greater for males $(4.8 ; 95 \%$ CI $2.4-9.6)$ than females. There was no difference in the duration of stay in hospital between the Black and White adolescent groups, with a mean of 17 weeks (s.d. $=19.8 ; 95 \%$ CI 9.0-26.6) for White and 18 weeks (s.d.=15.2; 95\% CI 9.3-26.9) for Black adolescents. The data on the duration of stay were available for 46 of 55 young people with psychotic disorders.

The overrepresentation of Black adolescents in the psychotic group was mainly due to the high numbers of adolescents of African origin, who made up $20 \%(11 / 55)$ of all adolescents with psychosis. However, only one of the eleven African youngsters had a diagnosis of schizophrenia, with the rest having a psychosis not otherwise specified or an affective psychotic disorder. On the other hand, six of the eight adolescents in the other Black groups (Black British, Black Caribbean) had schizophrenia, with only two having a psychosis not otherwise specified or an affective psychotic disorder. This diagnostic difference in the two groups was statistically significant (Fisher's exact test: $P=0.0063$ ).

Young refugees were highly represented in our sample of youngsters admitted for psychotic illnesses. There were 10 refugees in the sample of 55 young people. It is striking that eight of those ten were Black African adolescents. Among the Black group, largely those from an African background, there were high levels of social and family adversity, with many experiencing recent migration, poverty and not living with family.

On admission, 4 (16\%) of 25 White, 12 $(63 \%)$ of 19 Black, $1(14 \%)$ of 7 Asian and $1(25 \%)$ of 4 young people of other ethnic background were detained under the
Mental Health Act 1983. Black adolescents were significantly more likely to be detained on admission compared with their White counterparts ( $\chi^{2}$ test: $P<0.03$ ), but they did not seem to have a greater history of offending or more history of threatening or violent behaviour. However, there is no significant difference between the ethnic groups when detention at any point during the admission is taken into account: 13 $(52 \%)$ of 25 White, $15(79 \%)$ of 19 Black, $5(71 \%)$ of 7 Asian and $1(25 \%)$ of 4 adolescents of other ethnic background were detained at some point during the admission.

\section{DISCUSSION}

Our study showed overrepresentation of Black adolescents in the group of psychiatric in-patients with psychosis. The adolescents of African origin were particularly highly represented but were less likely to suffer from schizophrenia. Most of them had a refugee background and a high level of other adversities. Our findings are in keeping with the research in adult psychiatry, which found overrepresentation of Black patients in in-patient facilities because of psychosis (Koffman et al, 1997). Koffman et al (1997) showed that over four times as many Black as White adults, relative to the London population, were in-patients in London psychiatric units. However, although the adult research suggests that the rates of psychosis are increasing in the second generation of migrants from the Caribbean islands (Harrison et al, 1988), in our study the majority of the youngsters of African and Caribbean origin who were in-patients had arrived in this country over the past 10 years.

More adolescents from the Black group than the White group were detained under the Mental Health Act 1983 on admission, but that difference diminished during admission. One recent study of adult patients showed that detentions in England are over six times more likely to be of Black than of White people (Davies et al, 1996). Earlier studies showed that Black adult patients were more likely to experience a less desirable pattern of access to psychiatric services, particularly through police contacts (Dunn \& Fahy, 1990). However, a more recent study in South London concluded that adverse pathways to psychiatric care in African-Caribbean patients are not present at their first admissions but are likely to develop over time (Burnett et al, 1999).

\section{Reliability of findings}

This is the first study in the UK investigating links between psychiatric admission for psychosis and ethnic background in the adolescent population. Complete data were obtained for more than $90 \%$ of young people, but baseline demographic details were available for all the adolescents.

There was a high level of agreement on the diagnostic measures, including high interrater reliability for assigning the diagnosis, between clinical diagnoses and those assigned using OPCRIT and between diagnoses assigned by two raters of OPCRIT, one of whom was blind to ethnicity and clinical diagnosis. There was complete agreement between OPCRIT diagnoses and diagnoses established with psychosis using K-SADS.

\section{Explanation of findings}

Possible explanations for the findings are that the poorer social support and high level of adversities for the African adolescents with psychosis resulted in a greater need for admission. Another plausible explanation is that there is a higher incidence of psychosis in ethnic minority adolescents. Both of these may occur in association with a high level of exposure to stressors, including war and organised violence. It is relevant that post-traumatic stress disorder may occur comorbidly with non-schizophrenic psychoses (David et al, 1999). There may also be cultural variation in the experience of hallucinatory experiences among adolescents (Johns et al, 2002). A further possible explanation is that there has been diagnostic bias, but this is not likely in view of the care taken regarding diagnosis, the level of impairment of the psychotic group and similar duration of in-patient treatment prior to the survey date for the Black and White groups. 


\section{Limitations}

There are limitations to the sample methods that we used in the study. Because this was a cross-sectional survey of in-patients on one particular day, there is a possibility that those patients who had a longer duration of stay up until the study day would be overrepresented in the results and those with a shorter duration underrepresented. However, against this was the finding that the length of admission prior to the study day was not significantly different between the Black and White adolescent psychotic groups. Other limitations of the study include the small sample size. There was reliance on case-note data rather than the use of standardised interviews with the adolescents and informants to assess psychiatric state and ethnic and cultural background.

\section{Implications}

The current findings have implications for service planning with regard to the need for adolescent psychiatric beds and associated out-patient services. Areas with high numbers of ethnic minority populations, especially refugees, will need a higher level of psychiatric bed provision than areas with a more settled, White British population. Further research should investigate further the reasons for the ethnic variation in admission for adolescents, and whether there are variations in the incidence of adolescent psychosis and the pathways to care for this group.

\section{ACKNOWLEDGEMENTS}

We thank all the adolescents who participated in the study, as well as the psychiatrists and all the other health professionals who helped us with the study.

\section{REFERENCES}

American Psychiatric Association (1994) Diagnostic and Statistical Manual of Mental Disorders (4th edn) (DSM-IV). Washington, DC: APA.

Audini, B. \& Lelliott, P. (2002) Age, gender and ethnicity of those detained under Part II of the Mental Health Act 1983. British Journal of Psychiatry, I80, 222-226.

Burnett, R., Mallett, R., Bhugra, D., et al (1999) The first contact of patients with schizophrenia with psychiatric services: social factors and pathways to care in a multi-ethnic population. Psychological Medicine, 29, 475-483.

Chambers, W. J., Puig-Antich, J., Hirsch, M., et al (1985) The assessment of affective disorders in children and adolescents by semi structured interview. Testretest reliability of the schedule for affective disorders and schizophrenia for school-age children, present episode version. Archives of General Psychiatry, 42, 696-702.

\section{CLINICAL IMPLICATIONS}

- This study indicates that there is an overrepresentation of African and AfricanCaribbean adolescents with psychosis in in-patient psychiatric facilities.

- The rates of detention under the Mental Health Act 1983 are increased in young people of African and Caribbean origin, especially at the point of admission to hospital.

- Among the adolescent psychiatric in-patient population there is a significant number of young refugees with psychotic disorders who have experienced high levels of adversity.

\section{LIMITATIONS}

- The number of participants in each ethnic group was relatively small and broader ethnic categories had to be used for data analysis.

Background information was collected from the case notes or case summaries.

- The length of stay in hospital might affect the size of the difference between the ethnic groups because of the cross-sectional approach to the sampling.

JOVANKA TOLMAC, MD, MATTHEW HODES, PhD, Academic Unit of Child and Adolescent Psychiatry, Imperial College London

Correspondence: Dr Matthew Hodes, Academic Unit of Child and Adolescent Psychiatry, Imperial College London, St Mary's Campus, Norfolk Place, London W2 IPG. Tel: +44 (0)20 7886 II45; Fax: +44(0)2078866299; e-mail: m.hodes@imperial.ac.uk

(First received 25 July 2003, final revision 12 January 2004, accepted I5 January 2004)

Commander, M. J., Dharan, S. P., Odell, S. M., et al (1997) Access to mental health care in an inner-city health district. I: Pathways into and within specialist psychiatric services. British Journal of Psychiatry, $\mathbf{1 7 0}$ 312-316.

Craddock, M., Asherson, P., Owen, M. J., et al (1996) Concurrent validity of the OPCRIT diagnostic system. Comparison of OPCRIT diagnoses with consensus bestestimate lifetime diagnoses. British Journal of Psychiatry, 169, 58-63.

David, D., Kutcher, G. S., Jackson, E. I., et al (1999) Psychotic symptoms in combat-related posttraumatic stress disorder. Journal of Clinical Psychiatry, 60, 29-32

Davies, S., Thornicroft, G., Leese, M., et al (1996) Ethnic differences in risk for compulsory psychiatric admission among representative cases of psychosis in London. BMJ, 312, 533-537.

Dunn, J. \& Fahy, T. A. (1990) Police admissions to a psychiatric hospital. Demographic and clinical differences between ethnic groups. British Journal of Psychiatry, 156, 373-378.

Goodman, R. \& Richards, H. (1995) Child and adolescent psychiatric presentations of secondgeneration Afro-Caribbeans in Britain. British Journal of Psychiatry, 167, 362-369.

Harrison, G., Owen, D., Holton, A., et al (1988) A prospective study of severe mental disorder in AfroCaribbean patients. Psychological Medicine, 18, 643-657.

Hollis, C. (2000) Adult outcomes of child and adolescent-onset schizophrenia: diagnostic stability and predictive validity. American Journal of Psychiatry, I57, 1652-1659.
Johns, L. C., Nazroo, J.Y., Bebbington, P., et al (2002) Occurrence of hallucinatory experiences in a community sample and ethnic variations. British Journal of Psychiatry, 180, 174-178.

Koffman, J., Fulop, N. J., Pashley, D., et al (1997) Ethnicity and use of acute psychiatric beds: one-day survey in North and South Thames regions. British Journal of Psychiatry, I7I, 238-24I.

Lelliott, P., Audini, B. \& Duffett, R. (200I) Survey of patients from an inner-London health authority in medium secure psychiatric care. British Journal of Psychiatry, 178, 62-66.

Maden, A., Friendship, C., McClintock, T., et al (1999) Outcome of admission to a medium secure psychiatric unit. 2. Role of ethnic origin. British Journal of Psychiatry, 175, 317-321.

McGovern, D. \& Cope, R. (1987) First psychiatric admission rates of first and second generation AfroCaribbeans. Social Psychiatry, 22, 139-149.

McGuffin, P., Farmer, S. \& Harvey, I. (199I) A polydiagnostic application of operational criteria in studies of psychotic illness. Development and reliability of the OPCRIT system. Archives of General Psychiatry, 48 764-770.

Pipe, R., Bhat, A., Matthews, B., et al (199I) Section 136 and the African/Afro-Caribbean minorities. International journal of Social Psychiatry, 37, 14-23.

Williams, J., Farmer, A. E., Ackenheil, M., et al (1996) A multicentre inter-rater reliability study using the OPCRIT computerized diagnostic system. Psychological Medicine, 26, 775-783. 